\title{
Análisis de la Estructura Jerárquica del Inventario de Conductas de la Función Ejecutiva en una Muestra de Estudiantes Universitarios
}

\author{
Hierarchical Structure Analysis of Behavior Rating Inventory of Executive Functions \\ Self-Report in a Sample of University Student's
}

\author{
Pamela Ramírez ${ }^{1}$, Claudia Paz Pérez-Salas ${ }^{2}$ y Alonso Ortega ${ }^{3}$
}

\begin{abstract}
Resumen
El BRIEF-SR (Behavior Rating Inventory of Executive Functions Self-Report Version) es un inventario de conductas que permite evaluar el funcionamiento ejecutivo en contextos reales. La presente investigación tuvo como objetivo principal conocer las propiedades psicométricas de este cuestionario a través de una aplicación colectiva a 240 estudiantes universitarios y evaluar la estructura jerárquica propuesta por el modelo teórico subyacente. El análisis factorial confirmatorio mostró un buen ajuste a los datos y los coeficientes de consistencia interna de las escalas fueron adecuados. Los resultados sugieren que la versión en español del BRIEF-SR es un instrumento válido y confiable para evaluar el funcionamiento ejecutivo en adolescentes chilenos con edades entre los 17 y 18 años.
\end{abstract}

Palabras clave: funciones ejecutivas, pruebas neuropsicológicas, confiabilidad, validez

\begin{abstract}
The BRIEF-SR (Behavior Rating Inventory of Executive Functions Self-Report Version) is a Behavior scale developed to assess executive functioning in real contexts. The aim of the present investigation is to estimate the psychometrics properties of this questionnaire in a sample of 240 undergraduate students and test the hierarchical latent structure proposed by its theoretical model. The confirmatory factor analysis showed good fit indices for a hierarchical latent structure, and the internal consistency coefficients were adequate. Results suggest that the Chilean version of the BRIEF-SR is a valid and reliable instrument for assessing executive functions in adolescents from 17 through 18 years old.
\end{abstract}

Keywords: executive functions, neuropsychological tests, reliability, validity

Este artículo contó con financiamiento CONICYT-PCHA/Doctorado Nacional/2016-21161449

\footnotetext{
${ }^{1}$ Magíster en Psicología, Departamento de Psicología Universidad de Concepción, Chile.

${ }^{2}$ Doctora en Psicología, Departamento de Psicología Universidad de Concepción, Chile. La correspondencia relativa a este artículo debe ser dirigida al e-mail: cperezs@udec.cl

${ }^{3}$ Doctor en Ciencias. Escuela de Psicología, Universidad Adolfo Ibáñez, Chile.
} 


\section{Introducción}

Luria fue el primer autor que asoció el funcionamiento ejecutivo a un conjunto de funciones reguladoras del comportamiento humano. Para Luria, las funciones intelectuales tienen dos componentes: a) Uno denominado intelecto estático o formal, que contempla aquellas capacidades que intervienen en actividades como la conceptualización, el juicio o el razonamiento y b) otro denominado intelecto dinámico, que abarca todas aquellas capacidades necesarias para solucionar cualquier tipo de problema intelectual (Luria, 1973). Estas capacidades se subdividen, a su vez, en otras tales como: a) Planteamiento de problemas, b) Construcción de hipótesis resolutivas, c) Ideación de estrategias para confirmar o desechar hipótesis y d) Elección de las tácticas adecuadas (Luria, 1973).

La primera definición formal de las funciones ejecutivas (FE) se atribuye a Lezak (1982), quien las conceptualiza como un conjunto de capacidades mentales que permiten llevar a cabo conductas eficaces, creativas y adaptadas socialmente. Dichas capacidades mentales se manifiestan como habilidades cognoscitivas que permiten desempeñar tareas tales como: a) Anticipación, b) Establecimiento de metas, c) Diseño de planes y programas, d) Inicio de actividades y operaciones mentales, e) Autorregulación y monitoreo de tareas, f) Selección de comportamientos y conductas, y g) Flexibilidad en el trabajo cognitivo y su organización en el tiempo y el espacio; de modo de obtener resultados eficaces en la resolución de problemas, particularmente ante situaciones novedosas.

Una revisión acabada sobre este constructo fue realizada por Tirapu-Ustárroz, García-Molina, Luna-Lario, Roig-Rovira y Pelegrín-Valero (2008a, 2008b), quienes refieren la existencia de distintas aproximaciones al estudio de las FE, entre las que se encuentran los modelos de sistema simple (Cohen \& Servan-Schreiber, 1992; Grafman, 1995), modelos de constructo único (Baddeley \& Hitch, 1994; Duncan, Emslie, Williams, Johnson, \& Freer ,1996) y los modelos de procesos múltiples (Fuster, 2002; Miller \& Cohen, 2001; Miyake, Friedman, Emerson,
Witzki, Howerter, \& Wager, 2000; Shimamura, 2000; Stuss, Shallice, Alexander, \& Pincton, 1995; Zelazo et al., 2003). Pese a esta amplia gama de modelos, de los cuales se derivan diversas teorías explicativas, la literatura especializada concuerda en considerar esta compleja función como un constructo psicológico no unitario, compuesto por procesos cognitivos diversos que se combinan de múltiples maneras para operar en diferentes situaciones (TirapuUstárroz et al., 2008a).

Las FE emergen tempranamente, pero no maduran sino hasta la adultez temprana. Su desarrollo es crítico para el éxito académico tanto desde la escuela como en la universidad, incluso controlando por niveles de logro inicial y coeficiente intelectual (Diamond, 2016; Filippetti \& Richaud, 2016). En relación con ello, Korzeniowski (2011) alude a tres periodos sensibles en el desarrollo de las áreas prefrontales asociados a cambios que se vinculan a la adquisición de comportamientos ejecutivos: a) una primera etapa, entre los 4 y 8 años de edad, b) una segunda etapa, entre los 10 y los 12 años y c) una tercera etapa, entre los 15 y los 19 años. A partir de lo anterior, es posible concluir que el desarrollo de las FE se inicia en la infancia y se desarrolla progresivamente a medida que el individuo se enfrenta a situaciones nuevas que implican la resolución de problemas y el uso de estrategias metacognoscitivas (Roselli, 2003).

Tradicionalmente, la evaluación de las FE se ha desarrollado en entornos altamente controlados (e.g., situaciones experimentales, evaluaciones clínicas) a partir de pruebas neuropsicológicas objetivas basadas en el rendimiento del sujeto (e.g., Test de Clasificación de Cartas de Wisconsin (Heaton, Chelune, Talley, \& Kay, 1997), Test de Colores y Palabras de Stroop (Golden, 1994), Torre de Londres (Culbertson \& Zillmer, 2001)), las que evidencian una alta validez interna, pero escasa validez ecológica y generalizabilidad a tareas y contextos de la vida cotidiana (Chan, Shum, Toulopoulou \& Chen, 2008; García-Fernández, González-Pienda, Pérez, Álvarez, \& Álvarez, 2014; Gioia \& Isquith, 2004). Por ello se han desarrollado escalas de evaluación del comportamiento que buscan evaluar el funcionamiento ejecutivo en situaciones reales o cotidianas (Chan et al., 2008). La versión de 
autoreporte del Inventario de conductas de la FE para adolescentes (Behavior Rating Inventory of Executive Function; BRIEF-SR) es un instrumento que refleja mayor validez ecológica, dado que es el sujeto quien autoevalúa su conducta y responde al inventario atendiendo a cómo sería su forma de actuar en contextos reales y cotidianos (Guy, Isquith, \& Gioia, 2004).

Este cuestionario ha probado ser una medida de las FE tan válida como las pruebas neuropsicológicas tradicionales (Boschloo, Krabbendam, Aben, de Groot, \& Jolles, 2014; Gioia \& Isquith, 2004; Guy et al., 2004). No obstante, la correspondencia entre ambos tipos de mediciones es aún un asunto de debate, tanto por las bajas correlaciones encontradas entre el rendimiento de adolescentes en pruebas de ejecución y las dificultades observadas en su funcionamiento diario, como por el bajo acuerdo logrado entre los informantes al evaluar el comportamiento cotidiano de éstos (GarcíaFernández, Castro-González, Areces, FernándezCueli, \& Rodríguez-Pérez, 2014; Musso, 2009; Rubiales, 2014). Sobre la escasa correspondencia observada entre las mediciones y el funcionamiento diario de las personas Lezak, Howieson, Bigler y Tranel (2012) argumentan que ésta podría atribuirse a las propias condiciones en que se aplican las pruebas de ejecución, donde su alto grado de control experimental difiere considerablemente de los contextos naturales en los que los adolescentes se encuentran inmersos. Por otra parte, el escaso acuerdo observado entre los informantes podría explicarse por las diferencias del comportamiento en distintos contextos y situaciones. Pese a las limitaciones señaladas, García-Fernández et al. (2014) sostienen que la evidencia científica sugiere la necesidad de "explorar todo el rango de componentes que forman parte de las funciones ejecutivas... atendiendo tanto a los correlatos cognitivos, como los afectivos y conductuales de este constructo" (p. 220), lo que permitiría captar de mejor manera la naturaleza del funcionamiento ejecutivo en todas sus dimensiones.

La versión original del Behavioral Rating Inventory of Executive Function (BRIEF) (Gioia, 2000; Gioia, Isquith, Guy, \& Kenworthy, 2000) fue diseñada como una escala de reporte del funcionamiento ejecutivo de niños y adolescentes en edad escolar (5 a 18 años). El reporte es desarrollado por padres y profesores, quienes contestan los items del inventario en función de la conducta observada en los adolescentes. Los resultados se agrupan en dos índices principales: a) Regulación de la conducta (Behavior Regulation) y b) Metacognición (Metacognition); además de un Indice Global Compuesto (Global Executive Composite). Los valores normativos fueron obtenidos de una muestra de 2.139 protocolos completados por padres $(\mathrm{N}=1.419)$ y profesores $(\mathrm{N}=720)$ de sectores urbanos, suburbanos y rurales de Estados Unidos. Las escalas del BRIEF evidenciaron una consistencia interna apropiada y altos índices de validez y confiabilidad, lo que permite efectuar inferencias adecuadas sobre los comportamientos de los sujetos a partir de las puntuaciones observadas en este cuestionario (Messick, 1989).

El BRIEF ha demostrado constituir un buen recurso clínico para detectar dificultades ejecutivas en poblaciones diversas como, por ejemplo, en población con trastorno por déficit atencional con hiperactividad (TDAH), trastornos del espectro autista (TEA) (Guy et al., 2004), niños con parálisis cerebral (PC) (Bottcher, Meulengracht, \& Uldall, 2010), niños con discapacidad intelectual (DI) (Memisevic \& Sinanovic, 2014), y adolescentes con desorden bipolar (Passarotti, Trivedi, \& Patel, 2016).

Con posterioridad se han desarrollado otras versiones del BRIEF, en función de los rangos etarios de población. Hoy existen tres adaptaciones del inventario original, a saber, el BRIEF-P (versión para preescolares; Gioia, Espy \& Isquith 2002), el BRIEF-SR (versión de autoreporte para adolescentes; Guy et al., 2004), y el BRIEF-A (versión para adultos; Roth, Isquith, \& Gioia, 2005). La versión de autoreporte (BRIEF-SR) se desarrolló sobre la base de un modelo de procesos múltiples, donde la $\mathrm{FE}$ se conceptualiza como un constructo multidimensional que permite separar procesos o componentes, aun cuando se entiende que funcionan de manera integrada (Gioia, Isquith, Retzlaff, \& Espy, 2002). Esta integración de diversas dimensiones del funcionamiento ejecutivo permite al individuo desarrollar una conducta adaptada socialmente a través de la anticipación y el establecimiento de metas, la 
formación de planes, el inicio de las actividades, su autorregulación y la habilidad para llevarlas a cabo eficientemente (Tirapu-Ustárroz et al., 2008b). De acuerdo con esta aproximación teórica, los procesos cognitivos que forman parte de las FE son: a) Memoria de trabajo, b) Iniciativa, c) Planificación/organización, d) Monitoreo, e) Cambio, f) Inhibición, g) Autorregulación, h) Control emocional y i) Resolución de tareas, y dan origen a las escalas del BRIEF-SR (Guy et al., 2004).

Diversos estudios han tenido como propósito evaluar la estructura factorial subyacente al BRIEF (Arango, Puerta, \& Pineda, 2008; Egeland \& Fallmyr, 2010; García-Fernández, GonzálezPienda, et al., 2014; Gioia et al., 2000; Gioia et al., 2002; Huizinga \& Smidts, 2010; Roth, Lance, Isquith, Fischer, \& Giancola, 2013). Los hallazgos reportados sobre su estructura latente son disímiles, lo que podría atribuirse a las diferentes técnicas analíticas empleadas para estudiar su composición factorial (Pérez-Salas, Ramos, Oliva, \& Ortega, 2016). Por ejemplo, Gioia, Isquith, Guy y Kenworthy (2000) usaron la técnica de análisis factorial confirmatorio (AFC) en las versiones para profesores y para padres del BRIEF, encontrando en ambos casos evidencia para la existencia de dos factores de primer orden (i.e., Regulación de la conducta y Metacognición). Sin embargo, en ninguno de los dos análisis se obtuvo evidencia a favor de la existencia de un Indice Global Compuesto a la base del BRIEF (Gioia et al., 2000). Posteriormente, Gioia, Isquith, Retzlaff y Espy (2002), condujeron otro AFC en una muestra clínica utilizando la versión para padres del BRIEF. Los hallazgos no pudieron confirmar la estructura de dos factores de primer orden, ni tampoco evidencia sobre la existencia de un factor de segundo orden (i.e., Indice Global Compuesto). A partir de tales hallazgos, Gioia et al. (2002), evaluaron cuatro modelos diferentes, encontrando evidencia para una estructura factorial compuesta por tres factores de primer orden (i.e., Regulación de la conducta, Metacognición y Regulación emocional), pero sin corroborar la existencia de un factor de segundo orden. Posteriormente Arango, Puerta y Pineda (2008) emplearon la técnica de análisis factorial exploratorio (AFE) y los resultados sugirieron la existencia de un único factor latente a la base de las escalas del BRIEF, que los autores denominaron "Sistema de supervisión conductual" y que podría ser considerado análogo al Indice Global Compuesto. Luego, Egeland y Fallmyr (2010) obtuvieron evidencia que ratificó los hallazgos previos de Gioia et al. (2002), corroborando la existencia de tres factores de primer orden (i.e., Regulación de la conducta, Metacognición y Regulación emocional), utilizando la técnica de AFC. Pese a lo anterior, tampoco aportaron evidencia a favor de la existencia de un Indice Global Compuesto (Pérez-Salas et al., 2016). Recientemente, los estudios de García-Fernández, Castro-González, Areces, Fernández-Cueli y Rodríguez-Pérez (2014) y de Huizinga y Smidts (2010) emplearon el método de análisis de componentes principales (ACP) para evaluar la estructura factorial subyacente al BRIEF. Sin embargo, esta técnica no es apropiada para evaluar modelos confirmatorios $\mathrm{y}$, menos aún, para testear estructuras de organización jerárquica (Chen, West, \& Sousa, 2006; Reise, Moore, \& Haviland, 2010).

Como se mencionó en el párrafo anterior, la diversidad de modelos propuestos y de técnicas analíticas empleadas podría, en cierta medida, dar cuenta de la heterogeneidad en los hallazgos reportados sobre la estructura latente a las escalas del BRIEF. No obstante, Roth, Lance, Isquith, Fischer y Giancola (2013) implementaron por primera vez la técnica de AFC de segundo orden para evaluar la estructura jerárquica a la base del BRIEF. Los resultados obtenidos por Roth et al. (2013) usando la versión de adultos del BRIEF mostraron un buen ajuste para el modelo compuesto por tres factores de primer orden (i.e., Regulación de la conducta, Metacognición y Regulación emocional) y un factor de segundo orden (i.e., Indice Global Compuesto). El hallazgo de Roth et al. (2013) fue recientemente replicado por Pérez-Salas et al. (2016), quienes implementando un AFC de segundo orden con la versión original del BRIEF obtuvieron una solución factorial jerárquica similar. En el mismo estudio, Pérez-Salas et al. (2016) implementaron la técnica de modelamiento bifactorial, que de acuerdo con Chen, West y Souza (2006) y Reise, Moore y Haviland (2010) permitiría testear de mejor manera la existencia de un Indice Global Compuesto a la base del BRIEF, que no se 
explicaría directamente por los factores de primer orden. En otras palabras, la evaluación del modelo jarárquico usando la técnica de modelamiento bifactorial permitiría la interpretación por separado de los factores de primer orden y del Indice Global Compuesto, lo que posee ventajas al momento de la interpretación clínica de los resultados.

Considerando que la versión en español del BRIEF-SR no se encuentra validada en Chile, el presente estudio tiene como objetivo evaluar las propiedades psicométricas de la escala en una muestra de estudiantes universitarios chilenos de primer año. Se pondrá especial énfasis en la evaluación de la estructura factorial subyacente a las escalas del BRIEF-SR para corroborar si ésta se ajusta al modelo teórico jerárquico propuesto por los autores.

\section{Método}

\section{Diseño}

Se realizó una investigación instrumental (Ato, López, \& Benavente, 2013), destinada a analizar las propiedades psicométricas del cuestionario BRIEF-SR (Guy et al., 2004) y evaluar su estructura factorial latente.

\section{Participantes}

La muestra de estandarización estuvo formada por 240 estudiantes de primer año de distintas carreras de la Universidad de Concepción, Chile ( $\mathrm{N}=194$, mujeres; $\mathrm{N}=46$, hombres). Sus edades fluctuaron entre los 17 años, 9 meses y los 18 años, 11 meses $(M=18.3 ; D T=3.71)$. Todos los participantes otorgaron su consentimiento informado previo a su participación y el estudio fue conducido en concordancia con los lineamientos de la Declaración de Helsinki (World Medical Association, 2009).

\section{Instrumento}

Behavior Rating Inventory of Executive Function, Self-Report (BRIEF-SR) (versión en español). El Inventario de Conductas de la Función Ejecutiva es un cuestionario de autoreporte diseñado para evaluar la $\mathrm{FE}$ en individuos entre 11 y 18 años. En la muestra de estandarización, la consistencia interna del índice global de funcionamiento ejecutivo fue alta $(\alpha=.96)$ y de moderada a alta la de las escalas clínicas ( $\left.\alpha^{\prime} \mathrm{s}=.72-.96\right)$ (Guy et al., 2004). Asimismo, los datos de validación indicaron que los resultados del BRIEF-SR están moderadamente correlacionados con la versión para padres de la misma escala (Walker \& D'Amato, 2006).

La decisión metodológica de emplear al BRIEF-SR (Guy et al., 2004) en vez del BRIEFA (Roth et al., 2005), se sustenta en que el contexto universitario es más coherente con el tipo de situaciones evaluadas por el BRIEF-SR (i.e., funcionamiento en el ámbito educacional), a diferencia del BRIEF-A que evalúa el ajuste, entre otras cosas, al ambiente laboral. El BRIEF-SR consta de 80 reactivos, que exploran ocho dimensiones del funcionamiento ejecutivo, correspondientes a las escalas del instrumento (i.e., Inhibición, Cambio, Control Emocional, Monitoreo, Memoria de Trabajo, Planificación/Organización, Organización de materiales y Resolución de tareas). Estas escalas se organizan en dos índices más amplios: a) Metacognición y b) Regulación de la conducta. A su vez, el BRIEF-SR permite obtener un índice global de funcionamiento ejecutivo, denominado Índice Global Compuesto (Guy et al., 2004).

\section{Procedimiento}

Se tomó contacto con la Editorial PAR ${ }^{\circledR}$ para solicitar el instrumento en su versión en Español y la autorización para su uso en el presente estudio. Antes de estimar las propiedades psicométricas del instrumento se realizó una entrevista cognitiva (Smith-Castro \& Molina, 2011; Willis, 2004) a cinco estudiantes universitarios seleccionados mediante un muestreo por conveniencia, para evaluar si las instrucciones de aplicación y los ítems del instrumento eran claros y comprensibles. Las entrevistas cognitivas fueron grabadas y complementadas con notas de campo que daban cuenta de la actitud de los participantes al contestar el BRIEF-SR. El análisis se inició con la clasificación de los reactivos en dos categorías: con problemas de comprensión y sin problemas de comprensión. Posteriormente, se identificó la/s palabra/s que generaban dificultades de comprensión y las sugerencias de los informantes para su reemplazo. Este mismo criterio se empleó 
para analizar las instrucciones del cuestionario. Lo anterior permitió determinar en los ítems algunas palabras poco frecuentes en el Español de Chile, las cuales fueron reemplazadas por otras con mayor frecuencia de uso (Tabla 1).

Tabla 1. Modificaciones a los reactivos de la versión en español del BRIEF-SR

\begin{tabular}{lcc}
\hline $\mathrm{N}^{\circ}$ ítem & $\begin{array}{c}\text { Palabra del reactivo } \\
\text { original }\end{array}$ & $\begin{array}{c}\text { Palabra por la } \\
\text { que se cambia }\end{array}$ \\
\hline 4 & Asignaciones & Tareas \\
6 & Reguero & desorden \\
11 & me estanco & me bloqueo \\
14 & coraje & ira \\
18 & maestro(a) & profesor(a) \\
23 & lloroso(a) & lloro \\
31 & escuela & universidad \\
51 & espejuelos & espejo \\
\hline
\end{tabular}

Una vez realizadas las respectivas modificaciones al inventario de acuerdo con lo hallado en la entrevista cognitiva, se realizó un pilotaje en 30 estudiantes de primer año de la Universidad de Concepción, seleccionados mediante un muestreo no probabilístico, por conveniencia para identificar reactivos que pudiesen resultar ambiguos o difíciles de interpretar, con el fin de garantizar la comprensión de todas las preguntas del cuestionario. Finalmente, la versión corregida del BRIEF fue aplicada a la muestra de estandarización $(\mathrm{N}=240)$. La aplicación se realizó en las aulas, durante los últimos 20 minutos de clase.

\section{Análisis de Datos \\ Estadísticos Descriptivos}

En primera instancia se obtuvieron estadísticos descriptivos, de tendencia central (i.e., Media) y de dispersión (i.e., Desviación Típica) para cada una de las escalas del BRIEF-SR en la muestra de estandarización $(\mathrm{N}=240)$, los que corresponden a los valores normativos para su interpretación en la población adolescente Chilena. Estos análisis fueron realizados usando el software estadístico SPSS, versión 19 (IBM SPSS, 2011).

\section{Propiedades Psicométricas}

Confiabilidad. La confiabilidad se estimó indirectamente a través de la estimación de la
Consistencia Interna del instrumento. Para ello se estimó el Coeficiente Alfa de Cronbach (Cronbach, 1951) para cada escala del BRIEF-SR. Estos análisis fueron realizados usando el software estadístico IBM SPSS, versión 19 (2011).

Validez. De acuerdo con Reise, Moore y Haviland (2010) las técnicas de análisis factorial confirmatorio (AFC) de segundo orden y de modelamiento bifactorial son las únicas opciones analíticas que permiten evaluar e identificar multidimensionalidad $\mathrm{y}$, simultáneamente, la existencia de un factor general. Tales modelos tienen una estructura denominada "jerárquica" y es la que ha sido propuesta por los autores del BRIEF-SR. Por su parte, Chen et al. (2006) comparten una visión similar al respecto.

Por tal motivo se consideró útil efectuar ambos procedimientos analíticos y evaluar el ajuste de cada modelo. Posteriormente, se efectuó una comparación entre el ajuste de ambos modelos mediante una prueba de Chi-cuadrado (i.e., comparación anidada), reportándose el tamaño del efecto de la diferencia de ajuste entre ambos modelos mediante el estadístico $\omega$ de Cohen (Cohen, 1988).

$\mathrm{El}$ análisis factorial confirmatorio (AFC) de segundo orden se adecua a situaciones donde existe un modelo teórico que proponga: a) una estructura factorial de primer orden y b) un factor de segundo orden (i.e., un factor general que subyace a los factores de primer orden) cuyas cargas factoriales hacia las escalas se encuentran mediadas a través de los factores de primer orden (Jennrich \& Bentler, 2012). En este caso, el modelo teórico propuesto para el BRIEF (Gioia et al., 2002) se adapta a la definición de AFC propuesta por Jennrich y Bentler (2012) y, por ende, se espera que los datos se agrupen en torno a dos factores de primer orden (i.e., Metacognición y Regulación de la conducta) y un factor de segundo orden (i.e., Indice global de funcionamiento ejecutivo).

Posteriormente, se efectuó un modelamiento bifactorial, cuya diferencia esencial con el AFC de segundo orden es que permite evaluar los efectos directos del factor general sobre las escalas y, simultáneamente, los efectos del factor general a través de los factores de primer orden (i.e., efecto mediado). En ese sentido, se puede afirmar que el 
AFC de segundo orden es un caso especial de modelamiento bifactorial (i.e., modelo restringido), donde las cargas factoriales directas desde el factor general hacia las escalas son eliminadas (Jennrich \& Bentler, 2012). De este modo, se asume que los modelos de segundo orden están anidados en los modelos bifactoriales (Yung, Thissen, \& McLeod, 1999). El uso del modelamiento bifactorial se justifica cuando existe interés tanto en los efectos de los factores de dominio específico (i.e., de primer orden), como en el efecto independiente del factor general (Chen et al., 2006; Harman, 1976; Holzinger \& Swineford, 1937).

La comparación anidada no sólo permite observar qué modelo evidencia mejor ajuste sino, además, evaluar si es posible la interpretación independiente de los factores de dominio específico (i.e., Metacognición y Regulación de la conducta) y del factor general (i.e., Indice Global Compuesto). En síntesis, ambos análisis están orientados a la estimación de la validez de constructo del instrumento. Tanto el AFC de segundo orden como el modelamiento bifactorial fueron conducidos usando el software MPlus 7.11 (Muthén \& Muthén, 2013).

\section{Resultados}

\section{Estadísticos Descriptivos}

La Tabla 2 resume los estadísticos de tendencia central y de dispersión obtenidos para la versión Chilena del BRIEF-SR. En particular se reporta el valor promedio $(M)$ y la desviación típica $(D T)$ para cada escala del instrumento.

Tabla 2. Estadísticos descriptivos para la versión chilena del BRIEF-SR

\begin{tabular}{lcc}
\hline Escala & $M$ & $D T$ \\
\hline Inhibición & 19.41 & 4.10 \\
Cambio & 16.54 & 3.14 \\
Control emocional & 16.79 & 4.17 \\
Monitoreo & 8.11 & 2.23 \\
Memoria de trabajo & 20.02 & 4.34 \\
Planificación/Organización & 21.26 & 4.57 \\
Organización de materiales & 11.42 & 2.88 \\
Resolución de tareas & 16.61 & 3.85 \\
\hline
\end{tabular}

\section{Propiedades Psicométricas}

Confiabilidad. Los Coeficientes de Consistencia Interna (Cronbach, 1951) para las escalas del BRIEF-SR son reportados en la Tabla 2. Los valores del coeficiente $\alpha$ de Cronbach del BRIEFSR fluctúan entre $\alpha=.69$ y $\alpha=.83$, lo que permite inferir altos niveles de confiabilidad de la prueba. Cabe recordar que el valor de $\alpha$ representa el límite inferior del nivel de precisión de la prueba (Cronbach, 1951).

Tabla 3. Coeficientes de consistencia interna para las escalas del BRIEF-SR

\begin{tabular}{lcc}
\hline Escala & $\begin{array}{c}\alpha \mathrm{de} \\
\text { Cronbach }\end{array}$ & $\begin{array}{c}\mathrm{N}^{\circ} \mathrm{de} \\
\text { elementos }\end{array}$ \\
\hline Inhibición & .79 & 13 \\
Cambio & .74 & 10 \\
Control emocional & .82 & 10 \\
Monitoreo & .69 & 5 \\
Memoria de trabajo & .83 & 12 \\
Planificación/Organización & .79 & 13 \\
Organización de materiales & .72 & 7 \\
Resolución de tareas & .83 & 10 \\
\hline
\end{tabular}

Validez. El análisis factorial de segundo orden mostró un ajuste adecuado a los datos $\left(\chi^{2}=52.34\right.$, $g l=19, p<.01 ; \mathrm{CFI}=.942 ; \mathrm{RMSEA}=.086$ [90\% C.I. .058 - .114]; SRMR=.042). El valor RMSEA es levemente superior a los estándares recomendados (i.e., RMSEA $=.06 \quad(\mathrm{Hu} \&$ Bentler, 1999); RMSEA=.07 (Steiger, 2007)). Sin embargo, el intervalo de confianza al $90 \%$ permite inferir que el modelo posee un buen ajuste. Las cargas de los factores de primer orden resultaron todas significativas y mayores a .591. Las cargas del factor de segundo orden hacia los factores de primer orden fueron .719 y .966 , respectivamente (ver Tabla 3). La Figura 1. muestra el diagrama de la estructura jerárquica de segundo orden subyacente a las escalas del BRIEF-SR.

El modelamiento bifactorial mostró un ajuste a los datos que resultó estadísticamente significativo $\left(\chi^{2}=37.60, g l=11, p^{<.01 ; C F I=.954 ; ~}\right.$ RMSEA $=.100 \quad[90 \% \quad$ C.I. $.066 \quad-\quad .136]$; SRMR=.026), aunque sus índices de ajuste son peores en comparación con el modelo factorial de segundo orden. El valor RMSEA también es mayor a los estándares recomendados (i.e., RMSEA=.06 (Hu \& Bentler, 1999); RMSEA $=.07$ (Steiger, 2007)). 
Tabla 4. Cargas factoriales para los factores de primer y segundo orden del BRIEF-SR

(Factores de Primer orden)

Escala/Factor del BRIEF-SR Metacognición Regulación de la conducta

\begin{tabular}{|c|c|c|}
\hline Memoria de Trabajo & $.782^{\mathrm{a}}$ & \\
\hline Planificación/Organización & .832 & \\
\hline Organización de Materiales & .591 & \\
\hline Resolución de Tareas & .777 & \\
\hline Inhibición & & .615 \\
\hline Cambio & & .638 \\
\hline Control emocional & & .612 \\
\hline Monitoreo & & .732 \\
\hline $\begin{array}{l}\text { Indice Global Compuesto } \\
\text { (Factor de Segundo Orden) }\end{array}$ & .791 & .966 \\
\hline
\end{tabular}

${ }^{\text {a }}$ Solución estandarizada

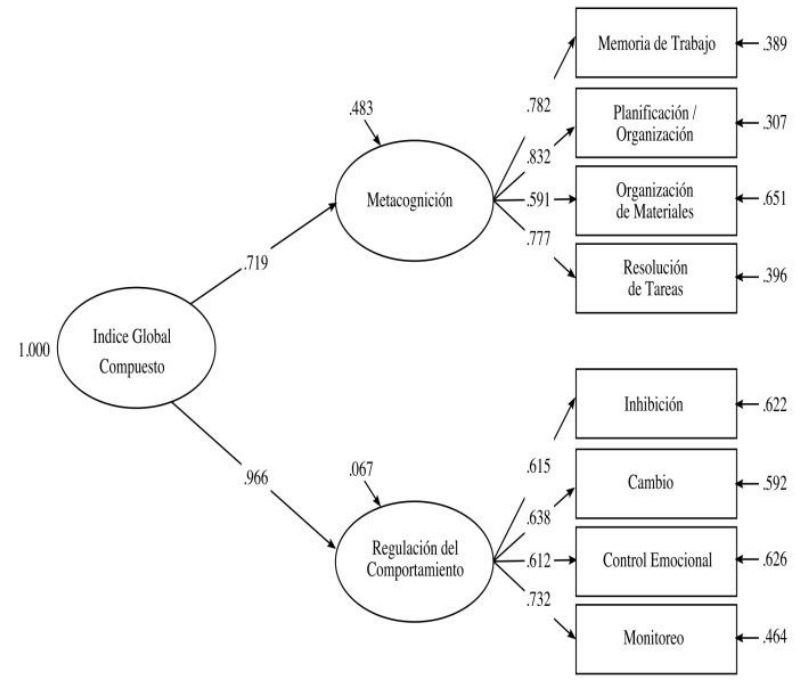

Figura 1: Diagrama de la estructura factorial de segundo orden subyacente a las escalas del

\section{BRIEF-SR}

Como se mencionó en la sección previa, se efectuó además una prueba de Chi-cuadrado para determinar cuál de los dos modelos posee un mejor ajuste a los datos. La comparación anidada muestra que la diferencia entre el ajuste de ambos modelos es no significativa (n.s.) lo que indica que estadísticamente ambos modelos poseen un buen ajuste a los datos $(\Delta \chi 2=14.74, \Delta$ g.l. $=8$, n.s. $)$. El tamaño del efecto para las diferencias entre ambos modelos se estimó mediante es estadístico Cohen's $\omega(\omega=.09)$ que de acuerdo a los estándares existentes puede ser considerada como pequeña (Cohen, 1988) (Tabla 4).

De ello se desprende que basta con el modelo más restringido para dar cuenta de la estructura latente a la base de las escalas del BRIEF-SR. Lo anterior se ajusta al principio de parsimonia, donde se asume que si dos modelos poseen un buen ajuste a los datos debemos conservar aquel que estima menos parámetros, es decir, el modelo más simple (Bentler \& Bonett, 1980; Kahn, 2006). En ese sentido, el modelo de segundo orden cumple con tal principio y, por ende, resulta ser un mejor modelo explicativo sobre la estructura factorial que subyace al BRIEF-SR.

\section{Discusión}

La naturaleza multidimensional de las FE hace que su evaluación sea un permanente desafío. A esta complejidad se suman las numerosas definiciones y variados procedimientos que existen para intentar determinar la naturaleza de sus componentes y la estructura de los mismos, no habiendo aún acuerdo entre investigadores (Jurado \& Rosselli, 2007).

El presente estudio tuvo como objetivo evaluar las propiedades psicométricas de la versión de autoreporte del Inventario de conductas de la FE para adolescentes (Behavior Rating Inventory of Executive Function; BRIEF-SR) (Guy et al., 2004), con especial énfasis en la evaluación de la estructura factorial subyacente para corroborar si ésta se ajustaba al modelo teórico jerárquico propuesto por los autores del instrumento.

Los resultados, entregan evidencia a favor de la estructura jerárquica a la base de a las escalas del BRIEF-SR, que resulta coherente con el modelo téorico propuesto por sus autores (i.e., dos factores específicos y un factor general) (Gioia et al., 2002) y con investigaciones recientes que analizan la estructura factorial del BRIEF empleando la técnica de análisis factorial confirmatorio (AFC) (Pérez-Salas et al., 2016; Roth et al., 2013).

De este modo, los datos expuestos en el presente artículo agregan evidencia que apoya una configuración jerárquica del cuestionario BRIEFSR (Pérez-Salas et al., 2016; Roth et al., 2013) y, por ende, de las FE evaluadas. Es decir, la presencia de dos factores latentes de primer orden (i.e., Regulación de la Conducta y Metacognición), más un factor latente de segundo 
Tabla 5. Prueba de chi-cuadrado de diferencia entre los modelos de segundo orden vs. bifactorial (comparación anidada)

\begin{tabular}{|c|c|c|c|c|c|c|c|c|}
\hline & \multirow[b]{2}{*}{$\chi^{2}$} & \multirow[b]{2}{*}{ g.l. } & \multirow[b]{2}{*}{ RMSEA (90\% C.I.) } & \multirow[b]{2}{*}{ SRMR } & \multirow[b]{2}{*}{ CFI } & \multicolumn{3}{|c|}{$\begin{array}{l}\text { Segundo orden vs. } \\
\text { bifactorial }\end{array}$} \\
\hline & & & & & & $\Delta \chi^{2}$ & $\Delta$ g.l. & $\omega$ \\
\hline $\begin{array}{l}\text { Modelo } \\
2^{\circ} \text { orden }\end{array}$ & $52.34 *$ & 19 & $.086(.058-.114)$ & .042 & .942 & $\begin{array}{l}14.74 \\
\text { (n.s.) }\end{array}$ & 8 & .09 \\
\hline $\begin{array}{l}\text { Modelo } \\
\text { bifactorial }\end{array}$ & $37.60 *$ & 11 & $.100(.066-.136)$ & .026 & .954 & & & \\
\hline
\end{tabular}

orden equivalente al Componente Ejecutivo Global. Esta configuración teórica es coherente con los hallazgos de Miyake et al. (2000) quienes estudiaron tres aspectos del funcionamiento ejecutivo (i.e., cambio, inhibición y actualización) concluyendo que, aunque son procesos diferentes, comparten elementos comunes, lo que podría homologarse a un sistema ejecutivo central. Así, basados en sus hallazgos, Miyake et al. (2000) señalan que las FE son constructos distintos pero correlacionados, sugiriendo además que estarían compuestos tanto por elementos "unitarios" (i.e., factores específicos) como "no unitarios" (i.e., factor global).

La naturaleza compuesta de las FE (i.e., componentes globales y específicos) también ha sido estudiada por Fisk y Sharp (2004) quienes mediante análisis factorial exploratorio replicaron la estructura de factores específicos propuesta por Miyake et al. (2000), agregándole un cuarto factor de acceso lexical que también sería parte de un sistema ejecutivo central.

Los resultados que hemos presentado en este artículo difieren de aquellos encontrados por Byerley y Donders (2013), cuyos datos no replicaron exactamente la estructura del BRIEFSR en una muestra de adolescentes con daño cerebral traumático, usando la misma técnica analítica. Pese a lo anterior, el estudio de Byerley y Donders (2013) evidenció un buen ajuste a los datos con AFC para la estructura que obtuvieron empleando análisis factorial exploratorio (AFE) la que, según señalaron, sería similar - aunque no igual - a la propuesta por Guy, Isquith y Gioia (2004).

Así, el aporte diferencial de la presente investigación fue testear de forma empírica el modelo teórico a la base del BRIEF-SR, mediante la técnica estadística del análisis factorial confirmatorio de segundo orden y modelamiento bifactorial, procedimientos que de acuerdo nuestro conocimiento, no habían sido utilizado antes para el análisis de este instrumento.

Los resultados tienen implicancias psicométricas y clínicas. En cuanto a las implicancias psicométricas, es posible señalar que existe evidencia empírica a favor del modelo teórico sobre FE propuesto por Gioia et al. (2002) y por ende, se otorga apoyo a la validez de constructo de este instrumento.

En cuanto a las implicancias clínicas, la existencia de un factor global subyacente a los factores específicos apoya la presencia de elementos comunes a las diversas FE y, por ende, a sus déficits, permitiendo también compensar dificultades en alguna función mediante la potenciación de otra, tal como Ramos y PérezSalas (2016) comprobaron en relación al Déficit de Atención con Hiperactividad, en donde a través de una intervención en la monitorización, se encontró una disminución del efecto negativo del déficit inhibitorio sobre la conducta de adolescentes (i.e., Efecto moderador de la monitorización en la relación entre el control inhibitorio y la conducta).

Específicamente, la evaluación del funcionamiento ejecutivo en la adolescencia, en población universitaria-no clínica, permitiría desarrollar planes de apoyo a los estudiantes durante los primeros años de universidad, toda vez que los procesos de maduración cerebral que comienzan en la temprana infancia se prolongan incluso hasta la post adolescencia (Arán, 2011). Dichos cambios se manifiestan a través de modificaciones estructurales y funcionales que dan cuenta de la emergencia de diversas funciones cognitivas (Casey, Giedd, \& Thomas, 2000). De este modo, la adolescencia puede ser considerada como un periodo del desarrollo en el cual es posible intervenir y/o potenciar el desarrollo de 
las FE, siendo esta una etapa donde la adquisición y desarrollo de comportamientos ejecutivos desempeñan un rol fundamental asociado al éxito/fracaso académico.

Considerando este aspecto, la presente investigación contribuye a validar el uso de un instrumento con validez ecológica que permite evaluar el funcionamiento ejecutivo de los estudiantes que ingresan a la universidad. En el contexto chileno, contar con un instrumento como el BRIEF-SR permitiría orientar el trabajo desarrollado por los centros de apoyo al estudiante, espacios que han surgido ante el aumento de la cobertura en educación superior y las dificultades que enfrentan los estudiantes al ingresar a la universidad.

Algunas limitaciones de este estudio dicen relación con la homogeneidad de la muestra (i.e., estudiantes universitarios de un rango restringido de edad), la desigualdad en cuanto al género y la falta de representatividad geográfica. No obstante, el tamaño muestral cumple con la relación de observaciones requeridas para la estimación de los parámetros que contempla el modelo evaluado (Kline, 2015). Estos aspectos disminuyen la posibilidad de generalizar los resultados a poblaciones diversas. Las limitaciones mencionadas, abren el desafío de ser incorporadas en investigaciones futuras para poder otorgar mayor posibilidad de generalizabilidad a los resultados obtenidos.

Es importante mencionar que la replicación de la estructura factorial jerárquica a la base del BRIEF en trabajos recientes (Pérez-Salas et al., 2016; Roth et al., 2013) provee un mayor entendimiento respecto de la naturaleza del funcionamiento ejecutivo y sus dimensiones subyacentes, lo que, puede ejercer ciertas implicancias para la práctica clínica, tal como se mencionó anteriormente. Por último, desde un punto de vista metodológico y psicométrico resulta importante destacar que la utilización de metodologías analíticas adecuadas (Chen et al., 2006; Reise et al., 2010) permite uniformizar y guiar, en cierta manera, el modo en que se evalúan modelos teóricos sobre funciones cognitivas que posean una estructura jerárquica a la base.

\section{Referencias}

Arán, V. (2011). Funciones ejecutivas en niños escolarizados: Efectos de la edad y del estrato socioeconómico. Avances en Psicología Latinoamericana, 29(1), 98-113. Recuperado de http://www.scielo. org.co/pdf/apl/v29n1/v2 9n1a08.pdf

Arango, O. E., Puerta, I. C., \& Pineda, D. A. (2008). Estructura factorial de la función ejecutiva desde el dominio conductual. Diversitas, 4(1), 63-77.

Ato, M., López, J. J., \& Benavente, A. (2013). Un sistema de clasificación de los diseños de investigación en psicología. Anales de psicología, 29(3), 1038-1059. doi:10.6018/ana lesps.29.3.178511

Baddeley, A. D., \& Hitch, G. A. (1994). Developments in the concepts of working memory. Neuropsychology, 8, 48493. doi:10.1037/0894-4105.8.4.485

Bentler, P. M., \& Bonett, D. G. (1980). Significance tests and goodness of fit in the analysis of covariance structures. Psychological bulletin, 88(3), 588. doi:10.1037/0033-2909.88.3.588

Boschloo, A., Krabbendam, L., Aben, A., de Groot, R., \& Jolles, J. (2014). Sorting Test, Tower Test, and BRIEF-SR do not predict school performance of healthy adolescents in preuniversity education. Frontiers in psychology, 5, 1-8. doi: 10.3389/fpsyg.2014.0 0287

Bottcher, L., Meulengracht, E. \& Uldall, P. (2010). Attentional and executive impairments in children with spastic cerebral palsy. Developmental Medicine \& Child Neurology, 52, 42-47. doi: 10.1111/j.14698749.2009.03533.x

Byerley, A. K., \& Donders, J. (2013). Clinical utility of the Behavior Rating Inventory of Executive Function-Self-Report (BRIEF-SR) in adolescents with traumatic brain injury. Rehabilitation Psychology, 58(4), 412. doi: 10.1037/a0034228.

Casey, B. J., Giedd, J. N., \& Thomas, K. M. (2000). Structural and functional brain development and its relation to cognitive development. Biological Psychology (54), 241 -257. doi: 10.1016/S0301-0511(00)00058-2 
Cohen, J. (1988). Statistical power analysis for the behavioural sciences. Lawrence Earlbaum Associates.: Hillsdale, New Jersey.

Cohen J. D., \& Servan-Schreiber D. (1992). Context, cortex, and dopamine: A connectionist approach to behavior and biology in schizophrenia. Psychological Review, 99, 45-77. doi:10.1037/0033295X.99.1.45

Cronbach, L. J. (1951). Coefficient alpha and the internal structure of tests. Psychometrika, 16(3), 297-334. doi:10.1007/BF02310555

Culbertson, W., \& Zillmer, E. (2001). The tower of London DX (TOL-DX) manual. North Tonawanda, NY: Multi-Health Systems.

Chan, R. C., Shum, D., Toulopoulou, T., \& Chen, E. Y. (2008). Assessment of executive functions: Review of instruments and identification of critical issues. Archives of clinical neuropsychology, 23(2), 201-216. doi: 10.1016/j.acn.2007.08.010

Chen, F. F., West, S. G., \& Sousa, K. H. (2006). A comparison of bifactor and second-order models of quality of life. Multivariate Behavioral Research, 41(2), 189-225. doi: $10.1207 / \mathrm{s} 15327906 \mathrm{mbr} 4102 \_5$.

Diamond, A. (2016). Why improving and assessing executive functions early in life is critical. En J.A. Griffin, P. McCardle, \& L.S. Freund (Eds.), Executive functions in preschool-age children: Integrating measurement, neurodevelopment, and translational research (pp. 11-43). Washington, DC: American Psychological Association.

Duncan, J., Emslie, H., Williams, P., Johnson, R., \& Freer, C. (1996) Intelligence and the frontal lobe: the organization of goal-directed behavior. Cognitive Psychology, 30(3), 257303. doi: 10.1006/cogp.1996.0008

Egeland, J., \& Fallmyr, Ø. (2010). Confirmatory factor analysis of the Behavior Rating Inventory of Executive Function (BRIEF): Support for a distinction between emotional and behavioral regulation. Child Neuropsychology, 16(4), 326-337. doi: 10.1080/09297041003601462

Filippetti, A., \& Richaud, MC. (2016). A structural equation modeling of executive functions, IQ and mathematical skills in primary students: Differential effects on number production, mental calculus and arithmetical problems. Child Neuropsychology, $8, \quad$ 1-25. doi: 10.1080/09297049.2016.1199665

Fisk, J. E., \& Sharp, C. A. (2004). Age-related impairment in executive functioning: Updating, inhibition, shifting, and access. Journal of Clinical and Experimental Neuropsychology, 26(7), 874-890. doi: 10.1080/13803390490510680

Fuster, J. (2002). Frontal lobe and cognitive development. Journal of Neurocytology 31, 373-385. doi: 10.1023/A:1024190429920

García-Fernández, Castro-González, P., Areces, D., Fernández-Cueli, M. S., \& RodríguezPérez, C. (2014). Funciones ejecutivas en niños y adolescentes: Implicaciones del tipo de medidas de evaluación empleadas para su validez en contextos clínicos y educativos. Papeles del psicólogo, 35(3), 215-223. Recuperado de http://www.papelesdelpsicolog o.es/vernumero.asp? $\mathrm{id}=2442$

García-Fernández, González-Pienda, J. A., Pérez, C. R., Álvarez, D., \& Álvarez, L. (2014). Psychometric characteristics of the BRIEF scale for the assessment of executive functions in Spanish clinical population. Psicothema, 26(1), 47-54. doi: 10.7334/psicothema2013.149

Gioia, G. A. (2000). BRIEF: Behavior rating inventory of executive function: Professional manual. Lutz, FL: Psychological Assessment Resources.

Gioia G. A., Espy K. A., \& Isquith, P.K. (2002). Behavior Rating Inventory of Executive Function, Preschool Version (BRIEF-P). Odessa, FL: Psychological Assessment Resources.

Gioia, G. A., \& Isquith, P. K. (2004). Ecological assessment of executive function in traumatic brain injury. Developmental neuropsychology, 25(1-2), 135-158. doi: 10.1080/87565641.20 04.9651925

Gioia, G. A., Isquith, P. K., Guy, S. C., \& Kenworthy, L. (2000). Test review behavior rating inventory of executive function. Child Neuropsychology, 6(3), 235-238. doi: 10.1076/chin.6.3.235.3152 
Gioia, G. A., Isquith, P. K., Retzlaff, P. D., \& Espy, K. A. (2002). Confirmatory factor analysis of the Behavior Rating Inventory of Executive Function (BRIEF) in a clinical sample. Child Neuropsychology, 8(4), 249257. doi: 10.1076/chin.8.4.249.13513

Golden, C. J. (1994). Stroop: Test de colores y palabras. Madrid: TEA ediciones.

Grafman J. (1995). Similarities and distinctions among current models of prefrontal cortical functions. Annals of the New York Academy of Sciences, 769, 337-368. doi:10.1111/j.17496632.1995.tb38149.x

Guy, S., Isquith, P., \& Gioia, G. (2004). Behavior Rating Inventory of Executive Function-Self Report Version (BRIEF-SR). Odessa, FL: Psychological Assessment Resources.

Harman, H. H. (1976). Modern factor analysis. Chicago, IL: University of Chicago Press.

Heaton, R. K., Chelune, G. J., Talley, J. L., \& Kay, G. G. (1997). WCST Test de clasificación de tarjetas de Wisconsin. Madrid: TEA Ediciones.

Holzinger, K. J., \& Swineford, F. (1937). The bifactor method. Psychometrika, 2(1), 41-54. doi: 10.1007/BF02287965

Hu, L. T., \& Bentler, P. M. (1999). Cutoff criteria for fit indexes in covariance structure analysis: Conventional criteria versus new alternatives. Structural equation modeling: A multidisciplinary journal, 6(1), 1-55. doi: 10.1080/10705519909540118

Huizinga, M., \& Smidts, D. P. (2010). Age-related changes in executive function: A normative study with the Dutch version of the Behavior Rating Inventory of Executive Function (BRIEF). Child neuropsychology, 17(1), 5166. doi: 10.1080/09297049.2010.509715.

IBM SPSS, S. (2011). IBM SPSS STATISTIC program, version 19 statistical software packages. New York, NY: IBM corporation,.

Jennrich, R. I., \& Bentler, P. M. (2012). Exploratory bi-factor analysis: The oblique case. Psychometrika, 77(3), 442-454. doi: 10.1007/s11336-012-9269-1

Jurado, M. B., \& Rosselli, M. (2007). The elusive nature of executive functions: A review of our current understanding. Neuropsychology review, 17(3), 213-233. doi:10.1007/s11065007-9040-z
Kahn, J. H. (2006). Factor analysis in counseling psychology research, training, and practice principles, advances, and applications. The counseling psychologist, 34(5), 684-718. doi: 10.1177/0011000006286347

Kline, R. B. (2015). Principles and practice of structural equation modeling. New York, NY: Guilford publications.

Korzeniowski, C. G. (2011). Desarrollo evolutivo del funcionamiento ejecutivo y su relación con el aprendizaje escolar. Revista de Psicología Universidad Católica de Argentina, 7(13),7-26. Recuperado de http://bibliotecadigital.uca.edu.ar/repositorio/r evistas/desarrollo-evolutivo-funcionamientoejecutivo.pdf

Lezak, M. D. (1982). The problem of assessing executive functions. International Journal of Psychology, 17(1-4), 281-297. doi: 10.1080/00207598208247445

Lezak, M. D., Howieson, D., Bigler, E., \& Tranel, D. (2012). Neuropsychological Assessment. New York: Oxford University Press.

Luria, A. (1973). Neuropsychological studies in the USSR. A review. I. Proceedings of the National Academy of Sciences, 70(3), 959964. Recuperado de https://www.ncbi.nlm.nih .gov/pmc/articles/PMC433397/

Messick, S. (1989). Meaning and values in test validation: The science and ethics of assessment. Educational researcher, 18(2), 511. doi: 10.3102/0013189X018002005

Memisevic, H. \& Sinanovic, O. (2014). Executive function in children with intellectual disability - the effects of sex, level and a etiology of intellectual disability. Journal of Intellectual Disability Research, 58(9), 830-837. doi: 10.1111/jir.12098

Miller E. K., \& Cohen JD. (2001). An integrative theory of frontal lobe function. Annual Review of Neuroscience, 24, 167-202. doi:10.1146/annurev.neuro.24.1.167

Miyake, A., Friedman, N. P., Emerson, M. J., Witzki, A. H., Howerter, A., \& Wager, T. D. (2000). The unity and diversity of executive functions and their contributions to complex "frontal lobe" tasks: A latent variable analysis. Cognitive psychology, 41(1), 49-100. doi:10.1006/cogp.1999.0734 
Musso, M. (2009). Evaluación de funciones ejecutivas en niños: Análisis y adaptación de pruebas en un contexto escolar. Revista Iberoamericana de Diagnóstico y Evaluación - e Avaliação Psicológica, 1(27), 157-178. Recuperado de http://www.aidep.org/03_ridep /R27/R278.pdf

Muthén, L., \& Muthén, B. (2013). Mplus 7.11. Los Angeles, CA: Muthén \& Muthén.

Passarotti, A., Trivedi N., \& Patel M. (2016) Executive function in adolescent bipolar disorder with and without ADHD comorbidity. Bipolar Disorder, 1, 101-108. doi:10.4172/jbd.1000101

Pérez-Salas, C. P., Ramos, C., Oliva, K., \& Ortega, A. (2016). Bifactor modeling of the Behavior Rating Inventory of Executive Function (BRIEF) in a Chilean Sample. Perceptual and motor skills, 122(3), 757-776.

Ramos, C., \& Pérez-Salas, C. (2016). Propiedades psicométricas: ADHD Rating Scale IV en formato autoreporte. Revista chilena de neuro-psiquiatría, $\quad 54(1), \quad$ 9-18. doi:10.4067/S0717-92272016000100002.

Reise, S. P., Moore, T. M., \& Haviland, M. G. (2010). Bifactor models and rotations: Exploring the extent to which multidimensional data yield univocal scale scores. Journal of personality assessment, 92(6), 544-559. doi: 10.1080/00223891.2010 .496477

Roselli, M. (2003). Maduracion cerebral y desarrollo cognoscitivo. Revista Latinoamericana de Ciencias Sociales, Niñez $y$ Juventud, 1(1), 25-144. Recuperado de http://www.scielo.org.co/scielo.php?script=sci _arttext\& pid=S1692-715X2003000100005

Roth, R., Isquith, P., \& Gioia, G. (2005). BRIEFA. Behavior Rating Inventory of Executive Function-Adult version professional manual. Lutz: Psychological Assessment Resources.

Roth, R., Lance, C. E., Isquith, P. K., Fischer, A. S., \& Giancola, P. R. (2013). Confirmatory factor analysis of the behavior rating inventory of executive function-adult version in healthy adults and application to attentiondeficit/hyperactivity disorder. Archives of clinical neuropsychology, 28(5), 225-234. doi: 10.1093/arclin/act031
Rubiales, J. (2014). Perfil ejecutivo en niños con trastorno por déficit de atención con hiperactividad. Revista Iberoamericana de Diagnóstico y Evaluación - e Avaliação Psicológica, 2(38), 31-54. Recuperado de http://www.aidep.org/03_ridep/R38/Art2.pdf

Smith-Castro, V., \& Molina, M. (2011). Cuaderno metodológico 5. La entrevista cognitiva: Guía para su aplicación en la evaluación y mejoramiento de instrumentos de papel y lápiz. San José CR: Instituto de Investigaciones Psicológicas, Universidad de Costa Rica.

Steiger, J. H. (2007). Understanding the limitations of global fit assessment in structural equation modeling. Personality and Individual differences, 42(5), 893-898. doi: 10.1016/j.paid.2006.09.017

Shimamura A.P. (2000). The role of the prefrontal cortex in dynamic filtering. Psychobiology, 28(2), 207-218. doi:10.3758/BF03331979

Stuss, D. T. Shallice, T., Alexander, M. P., \& Pincton, W. (1995). A Multidisciplinary Approach to Anterior Attentional Functions. Annals New York Academic of Science, 769, 191-211. doi: 10.1111/j.1749-6632.1995.tb3 8140.x

Tirapu-Ustárroz, J., García-Molina, A., LunaLario, P., Roig-Rovira, T., \& Pelegrín-Valero, C. (2008a). Modelos de funciones y control ejecutivo (I). Revista de neurología, 46(684), 92. Recuperado de http://www.neurologia.co $\mathrm{m} / \mathrm{sec} /$ resumen.php?id=2008119\#

Tirapu-Ustárroz, J., García-Molina, A., LunaLario, P., Roig-Rovira, T., \& Pelegrín-Valero, C. (2008b). Modelos de funciones y control ejecutivo (II). Revista de Neurología, 46(12), 742-750. Recuperado de http://www.neurolog ia.com/sec/resumen.php?id=2008252

Walker, J. M., \& D'Amato, R. C. (2006). Test review: Behavior Rating Inventory of Executive Function -Self- Report version. Journal of Psychoeducational Assessment, 24, 394-399. doi:10.1177/0734282906288390

Willis, G. B. (2004). Cognitive Interviewing: A tool for Improving Questionnaire Design: A Tool for Improving Questionnaire Design. Thousand Oaks, CA: Sage Publications.

World Medical Association. (2009). Declaration of Helsinki. Ethical principles for medical 
research involving human subjects. Journal of the indian medical association, 107(9), 403405.

Yung, Y. F., Thissen, D., \& McLeod, L. D. (1999). On the relationship between the higher-order factor model and the hierarchical factor model. Psychometrika, 64(2), 113-128. doi: 10.1007/BF02294531

Zelazo, P. D., Müller, U., Frye, D., Marcovitch, S., Argitis, G., Boseovski, J., et al. (2003). The development of executive function in early childhood. Monographs of the Society for Research in Child Development, 68(3),189. doi: 10.1111/j.1540-5834.2003.06803001 